trisnitrate of bismuth, three minims of diluted hydrocyanic acid, one drachm of tincture of columba, in half an ounce of mucilage of gum; four leeches to be applied over the tumour.

Third day.-Tenderness of the epigastrium slightly dimin ished; general uneasiness after eating also less, though the pain in the situation of the tumour is not so. In consequence of his complaining of lumbar pains, Mr. Whitfield, the resident medical officer, ordered a belladonna plaster to be applied to the loins, and half a grain of hydrochlorate of morphia at bedtime. The pain in the lumbar region was relieved, and less uneasiness was experienced after breakfast; tumour much less tender; tongue clean; appetite fair.

Eighth day. - Vomited his breakfast, consisting of coffee and toast, yesterday morning. In other respects the same.

Tenth day.-Prescription: a plaster of ammoniacum, with mercury, to be applied to the epigastrium, and five grains of conium pill every night; less pain after taking food.

Sixteenth day, - There being rather more pain than usual after taking food, Mr. Whitfield ordered a scruple of carbonate of magnesia in an ounce of peppermint-water. The epigastrium is rather more tender; there has been nausea, but no vomiting; bowels open; pulse quiet.

Seventeenth day.-Omit the mixture, and take the following:-Tincture of conium, one drachm; dilute hydrocyanic acid, three minims; carbonate of soda, ten grains: in mintwater three times a day. Also solution of hydrochlorate of morphia, half a drachm; aromatic spirit of ammonia, one drachm, in mint-water immediately.

Nineteenth day. - The patient has experienced much more pain and flatulence after taking food for the last few days, and for an argravation of which the above dranght was ordered. There is now some abdominal distension, but no increase; rather a diminution of tenderness on pressure. There has been no vomiting, but oceasionally a feeling of nausea. The bowels have required castor oil.

Twenty-third day.--Omit the mixture, and take the following draught every sixth hour: Ammonio-citrate of iron, four grains; dilute hydrocyanic acid, three minims, in mint water.

Twenty-sixth day. - Still very anæmic; the pain after taking food is much relieved, and the abdominal tenderness is also diminished; tongue clean; bowels regular; pulse 96

The patient lingered until June, 1853, when he died in great suffering. The post-mortem examination was conducted by Dr. Bristowe, demonstrator of anatomy to the hospital, and is described as follows:- Head not examined; chest, heart, and pericardium healthy; a few adhesions were found in both pleura; the lungs appeared perfectly sound, with the exception of a little muco-purulent fluid in the bronchial tubes. Abdomen. -On opening the abdomen, the left lobe of the liver was found to be adherent above to the diaphragm, and below to the stomach; the edge of this lobe immediately above the stomach was very hard and somewhat irregular, and the anterior part of the latter organ along the edge of the liver for about an inch downwards, was also hard, nodulated, and of a whitish colour; the liver and stomach appeared nearly continuous, and conld only be recognised by their difference of colour. The remainder of the anterior surface of the stomach appeared perfectly healthy. On lifting up the great omentum with the transverse colon, the portion of peritonæum passing from the latter to the spine was found protruded by an irregular mass of cancerous growth. The liver and stomach were now removed, and on opening the latter, its internal surface was found to present a large amount of disorganization; the cesophagus was healthy, as also all that part of the stomach to the left of the cesophageal opening, together with the greater part of the greater curvature and anterior surface; the remainder, however, consisting chiefly of the lesser curvature and the greater part of the posterior surface, was occupied by an ovoid cancerous ulcer about eight inches long, and five wide. This was bounded by tumid and overhanging edges of firmer scirrhous material, the thickness of which varied between half and three-quarters of an inch. The edge bounding the ulcer was abrupt, irregular, and undermined. The surface of the ulcer itself was anfractuons, and studded with projecting masses, some having the appearance of fungous granulations, and others of cancerous material in a state of destruction. It extended to and involved the pylorus, but not the duodenum; came also very near the osophageal opening, and though it can scarcely be said to have involved that orifice, still the tumid edge of the alcer obstructed it to a certain extent. On making a section through the left lobe of the liver into the stomach, the malignant disease was seen to hare spread from the latter into the former, so that by means of this deposit the two organs were continuous and adhering firmly. The cancer had the ordinary characters of scirrhus, firm, white, and somewhat glistening, and where it formed the floor of the ulcerated surface, it was in many places more or less softened and yellow. The liver was rather small, corrugated, and healthy, with the exception of the parts in the neighbourhood of the stomach. The other organs were healthy, the only circumstance worthy of notice being that the greater part of the mucous membrane of the duodenum and jejunum was much blackened. It thus appears that in this case, a strain first directed the attention of the patient to a tumour, which might have been existing a long time before the effort; it may also be remarked that blood was brought up from the stomach at a very early period; that the liver was affected by contiguity; that the ulcer was of considerable size, and that the pylorus became partially involved in the diseased action.

We now beg to direct attention to another case, of the same kind as the preceding, which was treated at Guy's Hospital.

\section{GUY'S HOSPITAL}

Malignant Disease of the Stomach.

(Under the care of Dr. GrLL.)

Wullam $\mathrm{P}$ _- aged forty-eight years, admitted May 18 th, 1853 , into Job ward. The patient is much emaciated, and his countenance anxious and haggard; he states that ho enjoyed good health until the middle of January last, (five months before admission, when he first complained of symptoms of indigestion, which lasted for several hours after taking food. These symptoms increased for a fortnight, when vomiting would occur about half an hour, or two hours, after taking food, the emesis senerally giving great relief. Since this time vomiting has always occurred about two hours after meals.

Present state. - Much emaciated, and romits soon after eating; at the pyloric end of the stomach is a hard trmour, whilst in the right lumbar and iliac regions a deep fossa is noticed. The corresponding regions, on the opposite side, are occupied by the stomach, the conformation of which can be easily recognised externally. The patient complains of constant thirst and loss of appetite; pulse 68 ; tongue furred, and covered with sordes; bowels relieved once in two or three days.

Dr. Gull ordered beef-tea, milk, with egg beat up in it, and six ounces of port-wine daily; chloric ether and morphine. Second day.-Continues about the same; vomiting after taking food as before. Passes but a few ounces of urine in the twenty-four hours.

Third day.--Is much weaker than yesterday; takes very little food besides wine; vomited matter is dark, and deposits a black mucous sediment. Dr. Gull examined the latter under the microseope, and diagnosed a malignant uleer of the stomach.

Fourth day.- Has had a restless night; taken nothing but a few teaspoonfuls of wine. The romited matter is now small in quantity and very thick; he complains of pain across the loins; tongue dry, and covered with portions of the vomited matter. He sank gradually, and died on the fifth day after admission.

Post-mortem examination, forty-eight hours after death.Body emaciated. Thorax: The left lung partially adherent; lungs, heart, and pericardium healthy. - Abdomen: The intestines shrunk and empty; stomach drawn down into the abdominal cavity; the pyloric extremity situated to the right and above the umbilicis. - Stomach : On opening the stomach a large fungating mass, highly injected, was found growing from the lesser curvature for an extent of three inches and a half from the pylorus, the latter being itself invaded by the growth. The latter was highly vascular, and the stomach and intestines contained a quantity of black digested blood, which tinged the walls. Liver and kidneys healthy.

The two preceding cases offer good examples of carcinomatous affection of the stomach, accompanied by great irritability of that carity, but without complete closure of the pyloric orifice, the patency of the intestinal canal having continued up to the fatal issue. But cases of cancer of the stomach are sometimes observed in which the pylorus becomes entirely closed, the vomiting being incessant, and very little communication existing between the stomach and bowels. These cases are really of a distressing description, and we shall just adduce one which some time ago was treated at St. George's Hospital, and noted by Dr. Barclay, medieal registrar to the institution. 